\title{
Respiratory precautions for MERS-CoV: acceptable risk-benefit determination
}

Li Yang $\underline{H s u^{1}}$, MBBS, MPH

As of May 29, 2014, there have been 636 laboratoryconfirmed cases of Middle East respiratory syndrome coronavirus (MERS-CoV), with 193 deaths, which translates into a case fatality rate of $30.3 \% .^{(1)}$ There is currently no specific treatment for the infection other than supportive medical care. The majority of recent cases appeared to arise from human-to-human transmission and have mainly occurred within the healthcare setting. ${ }^{(2)}$ Although Singapore has had no MERS-CoV cases to date, we remain at continued risk for MERS-CoV importation in view of the significant number of travellers to and from the Middle East for religious (i.e. Umrah and Hajj), recreational, medical and business purposes.

In this issue of Singapore Medical Journal, Chung et al have summarised a very interesting and relevant debate on whether surgical masks - as opposed to N95 respirators would suffice in the prevention of MERS-CoV transmission in non-aerosol-generating circumstances in the hospital setting. ${ }^{(3)}$ This debate was jointly organised in July 2013 by the Society of Infectious Disease (Singapore) and Infection Control Association (Singapore), with Chung et al updating the salient points with evidence published this year. ${ }^{(3)}$ Although no clear outcome was reached during the debate, it is noteworthy that in the ten months that have elapsed since the debate, there has been no new evidence supporting the use of the more expensive N95 respirator - which, incidentally, also impairs air exchange for the wearer ${ }^{(4)}$ and carries a fairly significant risk of adverse effects with prolonged use $\mathrm{e}^{(5,6)}$ - over the surgical mask in preventing MERS-CoV cross-infections.

Nonetheless, given the high case fatality rate of MERS-CoV and the lack of any specific treatment, it is understandable why healthcare staff and policymakers may prefer to support recommendations on the use of N95 respirators in preventing nosocomial MERS-CoV transmission, despite the increased costs and risk of adverse effects. What constitutes acceptable risks and benefits in this situation is therefore subjective and influenced by local perceptions and culture. While the use of masks or respirators is important in preventing the spread of such viruses, other infection control interventions (e.g. improving hand hygiene, and early detection and isolation of infected patients) are also important and additive in reducing the risk of MERS-CoV transmission. ${ }^{(7)}$

\section{REFERENCES}

1. Australian Government Department of Health. Middle East respiratory syndrome coronavirus (MERS-CoV): situation update 29 May 2014. Available at: http://www.health.gov.au/internet/main/publishing.nsf/ Content/ohp-mers-cov.htm/\$file/mers-cov-situpdate-20140529.pdf. Accessed May 30, 2014

2. World Health Organization. WHO risk assessment: Middle East respiratory syndrome coronavirus (MERS-CoV). Available at: http://www.who.int csr/disease/coronavirus_infections/MERS_CoV_RA_20140424.pdf?ua=1. Accessed May 30, 2014.

3. Chung SJ, Ling ML, Seto WH, Ang BS, Tambyah PA. Debate on MERS-CoV respiratory precautions: surgical mask or N95 respirators? Singapore Med J 2014; 55:294-7.

4. Lee HP, Wang de Y. Objective assessment of increase in breathing resistance of N95 respirators on human subjects. Ann Ocup Hyg 2011; 55:917-21.

5. Lim EC, Seet RC, Lee KH, et al. Headaches and the N95 face-mask amongst healthcare providers. Acta Neurol Scand 2006; 113:199-202.

6. Foo CC, Goon AT, Leow YH, Goh CL. Adverse skin reactions to personal protective equipment against severe acute respiratory syndrome - a descriptive study in Singapore. Contact Dermatitis 2006; 55:291-4.

7. Jefferson T, Del Mar CB, Dooley L, et al. Physical interventions to interrupt or reduce the spread of respiratory viruses. Cochrane Database Syst Rev 2011; (7):CD006207. 